\title{
Vanini en France: perspectives de recherche
}

\section{Marcella Leopizzi}

\section{Q OpenEdition}

\section{Journals}

\section{Édition électronique}

URL : http://journals.openedition.org/studifrancesi/3610

DOI : 10.4000/studifrancesi.3610

ISSN : 2427-5856

\section{Éditeur}

Rosenberg \& Sellier

\section{Édition imprimée}

Date de publication : 1 décembre 2012

Pagination : 505-512

ISSN : 0039-2944

\section{Référence électronique}

Marcella Leopizzi, «Vanini en France: perspectives de recherche », Studi Francesi [En ligne], 168 (LVI I III) | 2012, mis en ligne le 30 novembre 2015, consulté le 06 mars 2021. URL : http://

journals.openedition.org/studifrancesi/3610; DOI : https://doi.org/10.4000/studifrancesi.3610

\section{(c) $\odot \Theta \Theta$}

Studi Francesi è distribuita con Licenza Creative Commons Attribuzione - Non commerciale - Non opere derivate 4.0 Internazionale. 


\title{
DISCUSSIONI E COMUNICAZIONI
}

\author{
Vanini en France: \\ perspectives de recherche
}

Né dans les Pouilles, à Taurisano (Lecce), en 1585 et brûlé sur la place du Salin à Toulouse le 9 février 1619, Giulio Cesare Vanini a joué un rôle important dans la lutte pour la conquête de la liberté de pensée.

Docteur in utroque iure et carme sous le nom de Fra' Gabriele, dans ses deux œuvres l'Amphitheatrum ${ }^{1}$ et le De Admirandis', publiées respectivement à Lyon en 1615 et à Paris en 1616, Vanini a mis en discussion les certitudes de la tradition philosophique et théologique et a dénoncé les préjugés et les superstitions: il a, ainsi, ouvert de nouveaux horizons à la réflexion humaine.

Largement tributaires des nouvelles théories philosophiques naturalistes de l'École de Padoue - ville où il a vécu de 1608 à 1612 - et en particulier de la pensée de Pomponazzi, Cardano, Scaligero et Cremonini³ , ses idées concernant l'existence de Dieu, l'immortalité de l'âme, les miracles, les prières, la religion, l'éternité du monde, l'anthropocentrisme, la magie et l'astrologie ont trouvé un large écho chez les libertins français du XVII siècle.

Après l'amère et décevante expérience du séjour anglais (1612-1614) - pendant lequel, déjà en pleine crise sur le plan de la foi religieuse, d'abord il abjure le catholicisme mais, par la suite, il essaie d'obtenir le pardon papal -, il se rend à Paris où, dans un premier moment, il a des contacts directs avec le nonce Roberto Ubaldini. Il s'éloigne de l'Église catholique et cherche à s'insérer dans un milieu apte à le valoriser culturellement, à le protéger économiquement et à lui donner une collocation sociale prestigieuse $^{4}$. Par le biais de Thomas Dempster, un intellectuel écossais qu'il avait rencontré à Londres ${ }^{5}$, Vanini entre en contact avec Arthur d'Épinay de Saint-Luc abbé de Redon qui, à son tour, l'introduit dans les milieux de la Cour et le place sous la protection du maréchal François de Bassompierre ${ }^{6}$.

Le passage suivant, tiré de la dédicace à Bassompierre contenue dans le De Admirandis, exalte la beauté du maréchal et prouve que Vanini est entré en contact avec lui grâce à Arthur d'Épinay de Saint-Luc:

(1) Giulio Cesare Vanini, Amphitheatrum aeternæx providentix, Lyon, veuve de Antoine de Harsy, 1615.

(2) Giulio Cesare Vanini, De Admirandis naturæ reginx dexque mortalium arcanis, Paris, A. Perier, 1616.

(3) Cfr. Didier Foucault, Un philosophe libertin dans l'Europe baroque. Giulio Cesare Vanini (15851619), Paris, Champion, 2003, p. 167.

(4) Cfr. Francesco Paolo Raimond, «Vita di Giulio Cesare Vanini», p. 7, disponible sur le site internet suivant: http://www.comune.taurisano. le.it/citta_territorio/vanini.php. Cfr. aussi FRANCESCO DE PAOla, «Vanini in Francia: i confini di una presenza», in Il Seicento francese oggi, actes du colloque international de Monopoli (Bari), 27-29 mai 1993, "Quaderni del Seicento francese" dirigés par Giovanni Dotoli et Paolo Carile, Bari-Paris, Adriatica-Nizet, 1994, pp. 317-329.

(5) Ibid.

(6) Cfr. Francesco Paolo Raimondi, Mario Carparelli, Giulio Cesare Vanini. Tutte le opere, Milano, Bompiani, 2010, p. 307. 
Ego sane qui tot oberrans regiones, infinitas propemodum pulchritudinis vivas depictasque imagines conspexi, cum in quibusdam pictorum aedibus tuam aspexi effigiem (meam fatebor infidelitatem), «Pictoris»- dixi «hoc opus est, non Naturæ. Cum vero Illustrissimus Arcturus Espinoeus Sanlucius Rhedonum Abbas, omni laude sublimior ac Dominus meus multis nominibus colendissimus, in Excellentiae tuae praesentiam me introduxit, tam spectabili opificio obstupefactus, hausto tacito quodam divinitatis afflatu, immutatus videbar ita ut alia ex causa mendacij pictores redarguerim, utpote quia nec minimas tuae pulchritudinis partes adumbrarunt. Nobilissimum hoc formosissimumque corporis templum, divinum animae $\mathrm{Nu}$ men inhabitat, omnibus virtutibus genere praestantissimis, specie pulcherrimis, magnitudine praeclarissimis, excellentia ipsa admirandis circumseptum et communitum. Si Platonis essem alumnus, tanquam mundi animam te colorem atque deoscularer»?

Une affirmation contenue dans un autre passage du De Admirandis atteste que, pendant son séjour à Paris, Vanini jouit de la protection de Nicolas Brûlard, marquis de Sillery: «Esto, nam et nobilissimo Domino meo Nicolao Brulardo opinio haec

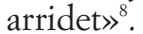

Aussi, pendant son séjour à Paris, Vanini commence-t-il à fréquenter des personnages importants et puissants appartenant aux milieux prestigieux de la noblesse française. Au fur et à mesure, il s'introduit dans le groupe des poètes et philosophes libertins qui se réunissaient autour de Théophile de Viau et qui jouissaient de la protection de Bassompierre, du duc Henri II de Montmorency et d'Adrien de Montluc comte de Cramail?.

C'est justement dans les milieux français qui vivaient un exceptionnel climat de liberté intellectuelle ${ }^{10}$ et où les premières lueurs du courant libertin commençaient à se manifester, que ses problèmes de foi et ses doutes vis-à-vis de la religion - qu'il nourrissait dès ses premiers contacts avec la patavina libertas - l'ont amené à l'athéisme et sont devenus, à leur tour, un point de repère considérable pour les "esprits forts" qui, pendant cette période-là, étaient de plus en plus tourmentés par une profonde crise religieuse et morale ${ }^{11}$.

À la suite de la découverte de l'Amérique, de la révolution copernicienne, du progrès des enquêtes scientifiques, les intellectuels à l'"esprit fort" commençaient à avoir une vision relativiste de tout le réel et à nourrir une sorte d'intellectualisme antichrétien ${ }^{12}$. La découverte de nouvelles terres habitées par des hommes aux mœurs profondément différentes les poussait à relativiser tout principe acquis et à mettre en crise la validité objective des religions ${ }^{13}$. En outre, la prise de conscience que der-

(7) Giulio Cesare Vanini, De Admirandis, cit., pp. VII-VIII.

(8) Vanini, De Admirandis, cit., p. 489. Cfr. RaImondi, Carparelli, Giulio Cesare Vanini. Tutte le opere, cit., p. 1816

(9) Cfr. Raimondi, Carparelli, Giulio Cesare Vanini. Tutte le opere, cit., p. 307. Cfr. aussi GIOvanni Dotoli, Temps de préfaces. Le débat théâtral en France de Hardy à la Querelle du «Cid», Paris, Klincksieck, 1996, pp. 127-128. Il est sans doute opportun de rappeler l'allusion à Vanini que l'on rencontre chez Théophile de Viau: dans l'Apologie de Théophile (in Euvres complètes, édition critique publiée par Guido SABA, Paris-Roma, Nizet-Edizioni dell'Ateneo, 1979, t. III, p. 74), il est question du père Garasse: «Je n'ay pas publié comme lui et Lucilio Vanino - dit Viau - les maximes des impies ...». Sur Théophile nous renvoyons à DAniela Dalla Valle, De Théophile à Molière, aspectos de una continuidad, Santiago de Chile, Editorial
Universitaria, 1968, t. I, pp. 50-99, et GUIDO SABA, Théophile de Viau: un poète rebelle, Paris, PUF, 1999.

(10) Cfr. Foucault, op. cit., p. 374.

(11) Cfr. Raimondi, Carparelli, Giulio Cesare Vanini. Tutte le opere, cit., pp. 119-120.

(12) Cfr. John STEPHENSON SpInK, La libre pensée française, Paris, Éditions Sociales, 1966, p. 17-20.

(13) Cfr. CeCILIA Rizza, Libertinage et littérature, Fasano, Schena, 1996, pp. 194-195. Cfr. aussi, de CECILIA RizzA, Barocco francese e cultura italiana, Cuneo, Saste, 1973. Sur le problème des rapports entre l'Europe et les nouveaux continents, nous renvoyons à LIONELLO SOzZI, Immagini del selvaggio. Mito e realtà nel primitivismo europeo, Roma, Edizioni di Storia e Letteratura, 2002: un chapitre y est consacré à la religion des primitifs. Plus en général, sur la pensée libertine, cfr. l'ouvrage classique de RENÉ PINTARD, Le libertinage érudit dans la première moitié du XVII siècle, Paris, Boivin, 1943. 
rière les désastres causés par les guerres de religion il y avait des intérêts qui allaient bien au-delà d'une foi religieuse sincère contribuait à augmenter leur mépris envers toute forme de dogmatisme. Qui plus est, les études des œuvres des grecs et des latins - qui, grâce à l'imprimerie se diffusaient désormais plus facilement et n'étaient plus soumises ni aux altérations des copistes ni aux tentatives des érudits de les interpréter à la lumière de la seule doctrine chrétienne - mettaient à disposition de leurs esprits critiques des contenus "nouveaux" qui bouleversaient les ipse dixit du passé.

Pendant ces années-là, parmi les œuvres qui ont contribué à la diffusion de ces thèses libertines, l'Amphitheatrum et notamment le De Admirandis de Vanini ont joué un rôle de tout premier plan. Dans ces ouvrages - tout particulièrement dans le deuxième -, notre philosophe met en discussion l'orthodoxie catholique en s'appuyant sur la pensée des philosophes anciens ainsi que sur l'interprétation matérialiste de la doctrine d'Aristote élaborée par l'École de Padoue. Il contribue ainsi à affirmer, voire renforcer, la pensée libertine qui était en train de se constituer et dont les sources thématiques principales s'appuient notamment sur la philosophie ancienne et l'aristotélisme padouan. À cause de sa portée transgressive, dès sa parution à Paris, le $1^{\text {er }}$ septembre 1616, le De Admirandis est au centre de discussions hardies et fait éclater un tel scandale que les autorités préposées à la censure ne restent pas longtemps indifférentes ${ }^{14}$. En effet, le $1^{\text {er }}$ octobre les deux censeurs qui avaient souscrit l'approbation ecclésiastique, Edmond Corradin et Claude Le Petit, soutiennent que le manuscrit qu'ils avaient approuvé était différent du texte imprimé. Par conséquent, ils demandent à la Faculté de Théologie de la Sorbonne d'émettre un décret de censure et exigent que ce décret soit enregistré dans le Livre des Conclusions «ut facilius impedire possent ne sub eorum approbatione amplius divulgentur» ${ }^{15}$.

Cet épisode marque profondément la vie de notre philosophe et, sans aucun doute, influe fortement sur son choix de quitter Paris. C'est probablement sur les conseils de ses amis, qu'il se rend à Toulouse sous le faux nom de Pomponio Usciglio.

Dès son arrivée dans cette ville, en 1617, Vanini fréquente assidûment le «Petit Louvre» d'Adrien de Monluc comte de Cramail et participe activement aux débats philosophiques qui ont lieu auprès de l'Académie des «Philarètes ou des Amoureux de la vertu» créée par ce comte ${ }^{16}$. Bientôt, à cause de ses discours libertins, il attire l'attention des milieux catholiques les plus intransigeants et ne va pas tarder à en subir les réactions ${ }^{17}$. Le 2 août 1618 il est arrêté par les Capitouls Paul Virazel et Jean Olivier $^{18}$. Le 9 février 1619, après un long procès dont malheureusement on ne possède pas les pièces, en l'accusant «d'athéisme» ${ }^{19}$ les deux Chambres - la Tournelle et la Grand'Chambre - du Parlement de Toulouse le condamnent à mort. Les pièces de ce procès n'ayant pas été retrouvées, les détails de la procédure de son arrestation et de sa condamnation restent obscurs et, en conséquence, beaucoup d'aspects concernant "l'affaire Vanini" sont encore à éclaircir.

Tout en s'enrichissant d'importantes tesselles, grâce aux études rigoureuses accomplies depuis quelques décennies par les spécialistes vaniniens, la mosaïque de la biographie de notre philosophe demeure tout de même incomplète, et elle le restera si les pièces du procès n'existent plus. Il faut espérer qu'elles sont encore "dans l'at-

(14) Cfr. Raimondi, Carparelli, Giulio Cesare Vanini. Tutte le opere, cit., p. 307.

(15) Ibid., p. 308.

(16) Ibid.

(17) Raimond, «Vita di Giulio Cesare Vanini», cit., p. 8 .

(18) Cfr. le registre des Annales de Toulouse gardé aux Archives Municipales de Toulouse sous la cote BB 269, année 1618, n. 182. Cfr. aussi le registre des Annales de l'Hôtel de ville de Toulouse (1618-1633) gardé aux Archives Municipales de cette ville sous la cote BB 278, année 1618, f. 13-14.

(19) Cfr. le manuscrit concernant l'arrêt de mort gardé aux Archives Départementales de la HauteGaronne de Toulouse, sous la cote B. N. 382, année 1619, p. 153 bis. 
tente" d'être retrouvées et qu'un jour elles seront récupérées. Il se peut, par exemple, qu'elles se trouvent dans les 80.000 sacs, conservés aux Archives Départementales de la Haute-Garonne de Toulouse, qui se rapportent à l'activité judiciaire toulousaine de la fin du XV ${ }^{\text {e }}$ siècle à la Révolution et qui n'ont pas encore été inventoriés. Si cette hypothèse se confirme, des documents inédits, relatifs à la fin tragique de notre philosophe, seront à la disposition de la communauté scientifique et enrichiront considérablement les études vaniniennes.

De même, à notre avis, de nouvelles perspectives de recherches, concernant la biographie de Vanini et en particulier les milieux qu'il a fréquentés pendant son séjour en France, pourraient s'ouvrir en analysant les notes manuscrites appartenant à Adolphe Baudouin, contenues dans le "fonds Baudouin" des Archives Départementales de la Haute-Garonne de Toulouse. Archiviste en chef de ces Archives de 1857 à 1895 et correspondant avec de nombreux bibliothécaires et érudits français et étrangers, Adolphe Baudouin a essayé, toute sa vie durant, de recueillir le plus de renseignements et de documents possible sur la biographie et l'œuvre de Vanini. Au fur et à mesure, il regroupait toutes ses recherches en «notes», auxquelles il attribuait un numéro et un titre, et, selon le sujet, il rassemblait toutes ces notes en sections.

Le "fonds Baudouin" contient toute la correspondance de Baudouin ainsi que toutes ces notes manuscrites que l'archiviste avait divisées en quatre sections et intitulées: Histoire critique de J.C. Vanini, Notes biographiques sur J.C. Vanini, Opinions de J-C. Vanini, Documents à consulter sur J.C. Vanini20. Ces notes concernent les informations et les documents que Baudouin possédait et qu'il a publiés seulement en partie étant mort avant de pouvoir achever l'œuvre monumentale que probablement il avait l'intention de rédiger. En conséquence, à notre avis, ce fonds représente une mine d'information pour les études vaniniennes non seulement en raison de ce qu'il contient mais surtout grâce aux perspectives de recherche qu'il offre. Selon nous, en effet, en s'appuyant sur les notes de Baudouin et en poursuivant et en développant les recherches qu'il avait entreprises ou bien qu'il s'était proposé d'entreprendre, l'on pourra parvenir à des découvertes importantes.

Dans ce but, nous allons donner quelques indications concernant les sujets traités dans ces notes et, par la suite, dans l'espoir d'ouvrir de nouveaux horizons aux études vaniniennes concernant le séjour de notre philosophe en France et ses rapports avec les milieux libertins, nous allons reporter la liste détaillée des notes contenues dans la quatrième section qui concerne justement les «documents à consulter» se référant aux rapports entre Vanini et d'autres personnages, dont la plupart sont des Français de son époque ${ }^{21}$.

Dans la première section - MSS 160: Histoire critique de J.C. Vanini-sont énumérées 359 notes manuscrites relatives à l'analyse des deux œuvres de Vanini et en particulier touchant aux pages de ces ouvrages qui contiennent des allusions autobiographiques; en outre, on peut lire les articles - en version manuscrite et sous forme imprimée - écrits par Baudouin et intitulés «Histoire critique de J.C. Vanini dit Lucilio», publiés dans la Revue philosophique de la France et de l'étranger ${ }^{22}$. L'on trouve aussi, pour terminer, quatre dossiers manuscrits dont voici les coordonnées:

(20) A posteriori on leur a attribué les cotes suivantes: MSS 160, MSS 161, MSS 162, MSS 163.

(21) Nous avons fourni l'inventaire complet du "fonds Baudouin" dans notre article: Giulio Cesare Vanini nei manoscritti di Adolphe Baudouin. La leggenda di Luciolo, in Humanitas et civitas. Studi in memoria di Luigi Crudo, par Giuseppe CARAMUSCIO et Francesco DE PaOla, "Quaderni de l'Idomeneo", Società di Storia Patria - sezione di Lecce, Galatina, Edizioni Panico, 2010, pp. 261-284.

(22) 1879, pp. 49-71, 157-178, 259-290, 387-408. 
1) Luigi Moschettini, «Vita di Giulio Cesare Vanini», Rivista europea, 1879, 16 mars, vol. XII, fasc. II, pp. 256-271;

2) Procés verbal du Synode Diocésain de Pasques du 16 avril 1619 faict et tenu par M.M. Jean de Rudele vicaire général de l'Archevesché;

3) épitaphe de Voltaire par le Père Papillon du Rivet, jésuite;

4) lettre adressée à Baudouin, écrite à Paris le 25 octobre 1879, de la part du secrétaire de la Revue historique (76, rue S. Assas), concernant le livre sur Vanini de Palumbo.

La deuxième section - MSS 161: Notes biographiques sur J.C. Vanini-contient 46 notes manuscrites relatives à la biographie de Vanini. On y trouve, entre autres, des renvois aux Histoires tragiques de François de Rosset; au Mercure François, 1619, tome V, p. 63-64; à la Doctrine curieuse de François Garasse; aux Quaestiones celeberrimae in Genesim de Marin Mersenne; à l'œuvre Patiniana et Naudæana; au Nouveau dictionnaire historique de Chauffepié; aux Mémoires d'Étienne de Malenfant; à l'Inventaire général des affaires de France depuis la mort de Henri le Grand jusqu'au dernier jour de l'an 1620 par D'Autreville; à l'Histoire générale des derniers troubles arrivés en France (1622) de Pierre Mathieu; et à l'Histoire de Louis le Juste par Scipion Dupleix, édition de 1635.

La troisième section - MSS 162 Opinions de J-C. Vanini - présente 30 notes manuscrites se rapportant à la pensée de notre philosophe vis-à-vis des miracles, des prières, des saints, des démons, du péché originel, le la résurrection des morts, de l'éternité du monde, de la mortalité de l'âme, de l'influence des astres, de la force de l'imagination, des rêves, et de l'influence de l'alimentation sur les mœurs.

La quatrième section - MSS 163: Documents à consulter sur J.C. Vanini-consiste en 47 notes manuscrites dont nous reportons ci-après tous les titres complets dans l'espoir que les informations contenues dans chacune de ces notes pourront mener les spécialistes vaniniens vers de nouvelles et fructueuses recherches:

note 1: concernant le nonce Ubaldini

note 2: chanceliers de France de 1607 à 1624

note 3: note sur François de Saint-Félix d'Aussargues procureur général

note 4: extrait de l'Histoire de l'édit de Nantes; extrait de la Biographie toulousaine; por-

trait de Gilles Le Mazuyer par l'annaliste Barthélemy de Gramond; extrait des Annales de l'Hôtel de ville de Toulouse

note 4 bis: extrait de Mézeray concernant l'assemblée des Notables

note 5: extrait de l'abrégé chronologique de l'Histoire de France sous le Règne de Louis

XIII pour servir de suite à celui de François de Mézeray

note 5 bis: extrait de l'Histoire Ecclésiastique de Fleury

note 6: extraits du Mercure françois 1615, tome III, p. 222 et tome IV, p. 65-76

note 7: extraits du Mercure de France concernant les craintes inspirées en Angleterre par

l'assassinat de Henri IV (1610); la conspiration des pauvres (1605-1606)

note 8: extraits du Mercure de France concernant les efforts du clergé pour faire admettre

en France le Concile de Trente

note 8 bis: extraits des actes du Concile de Trente

note 9: extraits du Mercure françois (et de Mézeray) concernant la répression des duels

note 10: livres ultramontains condamnés par le Parlement de Paris

note 11: extraits du Mercure françois concernant le poète Théophile

note 12: autorisation donnée à deux Docteurs en Théologie de Toulouse par la Congré-

gation des Cardinaux Inquisiteurs de lire des livres hérétiques

note 13: octobre 1618 concernant Louis de Lavalette fils du duc d'Epernon nommé ar-

chevêque de Toulouse à 23 ans sans avoir reçu les ordres

note 14: 1618-1619 séjour du P. Coton à Toulouse

note 15: chanoines de $\mathrm{S}^{\mathrm{t}}$ Etienne amis de $\mathrm{M}$. de Rudelle et des Jésuites

note 15 bis: 1621 époque de la mort de Monsieur Jean de Rudelle vicaire général 
note 16: 18 novembre 1621 séjour à Toulouse du Cardinal de Retz archevêque de Paris note 16 bis: novembre 1621 séjour de Louis XIII à Toulouse

note 17: extrait des Mémoires de Bassompierre

note 18: extraits des Mémoires de Sully

note 19: extraits des Mémoires de Richelieu

note 20: extraits de Pierre Bayle

note 21: concernant Arthur d'Espinay $\mathrm{S}^{\mathrm{t}} \mathrm{Luc}$ abbé de Rhedon

note 21 bis: ce que Vanini dit de Louis XIII et de Marie de Médicis

note 22: concernant Guillaume de Catel

note 23: extrait des délibérations du Chapitre $S$. Sermin

note 24: extrait du Mercure françois, 1619, tome V, p. 108 et suiv.

note 25: le comte de Caraman autrement dit le comte de Cramail, de Carmaing, de Garamain

note 26: le Sieur de Francon

note 27: la vie de Cour d'après Mathurin Régnier

note 28: chevalier de Sillery; lettres patentes du roi Louis XIII

note 29: préliminaires d'une enquête sécrète

note 30: feu Noalhes

note 31: extrait des Erotemata du P. Théophile Raynaud jésuite

note 32: greffiers commis à la garde des portes de la ville de Toulouse

note 33: arrêt du Conseil d'État contre le P. Pelissier doyen de la Faculté de Théologie

de Toulouse qui avait censuré un livre du Père Annat approuvé par la Sorbonne et muni d'un

Privilège du Roi

note 34: procédure en matière de monitoire

note 35: opinion de Voltaire sur Vanini

note 36: extrait des Mémoires d'Amelot de la Houssaye concernant les lettres d'abolition note 37: extrait des Mémoires d'Amelot de la Houssaye concernant les bénéfices

note 38: noms des cinq Présidents du Parlement de Toulouse en 1619

note 39: extrait du Ménagiana qui cite les Histoires tragiques de Rosset article Vanini

mail

note 40: extraits de l'Histoire généalogique du Père Anselme concernant le comte de Cra-

note 41: titres et approbations des ouvrages de Thomas Campanella (on y trouve la locution de Naturae arcanis)

note 42: extraits de l'Histoire de Louis XIII pour servir de suite à l'Histoire de Mézeray

note 43: note sur le Père Garasse

note 44: notes sur le Président Bertier

note 45: note sur Barthélemy de Gramond

note 46: extraits de La Rocheflavin relatifs au Parlement de Toulouse

note 47: extrait des recherches sur la Compagnie de Jésus du temps du P. Coton par le P. Prat, Lyon, 1876

Si, faute d'une documentation d'archives complète, certains aspects de la biographie de Vanini restent obscurs, par contre les caractéristiques de sa pensée sont bien connues car ses œuvres ont été largement étudiées par d'éminents spécialistes qui lui ont attribué un rôle crucial dans l'histoire de la libre pensée.

Fils du doute de Montaigne ${ }^{23}$, Vanini a été, répétons-le, l'un des plus importants coryphées du courant libertin ${ }^{24}$. Le bûcher qui a provoqué sa mort n’a pas éteint les

(23) Le doute montaignien est, en effet, à la base de tout travail critique de cette période-là car, en permettant de toujours chercher au-delà des vérités du moment, le «que sçay-je?» (Essais, II, 12, 527, B) devient le tremplin de la recherche de la vérité, voire l'antidote à tout dogmatisme Cfr. GIOvanNI
Dotoli, Les libertins et Montaigne, «Montaigne Studies», 2007, pp. 3-16.

(24) Cfr. Giovanni Papuli, «Approccio ai libertini», «Bollettino di storia della filosofia dell'Università degli studi di Lecce», 1996-2002, vol. XII, pp. 77-93. 
traces de son existence et encore moins celles de sa pensée. Par ses discours sur la cosmologie, la météorologie, la géologie, la physique, la botanique, la zoologie, la médecine, la psychologie et la religion, grâce auxquels, dès son vivant, il s'est inséré dans les débats culturels les plus en vogue à son époque, Vanini a continué à vivre même après sa mort. Ses opinions, transgressives et novatrices, ont eu un grand écho chez les libertins et ont contribué à jeter les bases de la philosophie moderne.

Vécu entre deux siècles, au moment du passage du climat culturel de la Renaissance aux instances de la pensée scientifique du XVII ${ }^{\mathrm{e}}$ siècle, Vanini a subi les contradictions typiques de son époque, c'est-à-dire d'une période de transition où le désir de liberté et le besoin de renouvellement s'opposaient à l'intolérance religieuse et à l'intransigeance politique et faisaient appel à l'opportunité de la dissimulation (il suffit de rappeler l'arrière-boutique de Montaigne ou la Dissimulazione onesta de Accetto). Par ses attitudes et ses ouvrages, il a inauguré la physionomie de l'écrivainphilosophe libertin qui, au nom de la raison, professe une totale liberté d'esprit, mais qui vit et écrit en dissimulant sa propre incrédulité.

Loin d'être un plagiat ${ }^{25}$, en effet, son œuvre réside sur un ensemble de citations vouées à donner à son discours un double sens apte à exposer les idées transgressives voilées par la simulation du respect des principes jusqu'alors admis. Cette duplicité, voire cette séparation profonde entre la dimension individuelle et la sphère collective est présente chez tous les libertins. D'ailleurs, dans l'ouvrage intitulé Naudæana, en parlant de Vanini, entre autres, Gabriel Naudé réfléchit sur les avantages dérivant de la maxime de Cremonini «intus ut libet, foris ut moris est»:

L'Italie est pleine de libertins et d'athées et de gens qui ne croyent rien, et neanmoins le nombre de ceux qui ont écrit de l'immortalité de l'ame est presque infini; mais je pense que ces mêmes écrivains n'en croyent pas plus que les autres; car c'est une maxime que je tiens pour certaine, que le doute qu'ils en ont est une des premieres causes qui les oblige d'en écrire, joint que tous leurs écrits sont si foibles que personne n'en peut devenir plus assuré; mais au contraire au lieu d'instruire ils sont propres à faire douter de tout. [...] J'ai été trois mois durant dans la conversation de Cremonini. [...] Ce Cremonini étoit grand Personnage, un esprit vif et capable de tout, un homme deniaisé et gueri du sot, qui sçavoit bien la verité, mais qu'on n'ose pas dire en Italie. Tous les Professeurs de ce païs-là, mais principalement ceux de Padoüe sont gens déniaisez, d'autant qu'étant parvenus au faite de la science, ils doivent étre détrompez des erreurs vulgaires des siècles et bien connoître l'opinion d'Aristote, de l'esprit duquel ce Cremonini est un vrai Tiercelet et parfait abregé. Ces Messieurs là sont gens raffinez, et dont le nombre est grand en Italie, sçavent bien discerner dans les grands, le vrai d'avec le faux. [...] Cremonini cachoit finement son jeu en Italie: nibil babebat pietatis, et tamen pius baberi volebat. Une de ses maximes étoit: intus ut libet; foris ut moris est. Il y en a bien en Italie qui n'en croyent pas plus que Cremonini, Machiavel et lui étoient à deux de jeu, et Epicure, Lucrece, Cardan, Castellanus, Pomponace, Bembe, et tous ceux qui ont écrit de l'Immortalité de l'Ame. Pline a été un des chefs. Vanini en son Amphitheatre dit: que c'est la grande Secte que celle des Athées, qui est grossie de la plûpart des Princes utriusque ordinis, et d'un grand nombre de sçavans anciens, comme Polybe, Ciceron, Cesar, Juvenal, Horace, Socrate, Homere, Euripide, Virgile, $\operatorname{etc}^{26}$.

(25) Cfr. Luigi Corvaglia, Le opere di Giulio Cesare Vanini e le loro fonti, Milano, 1933-1934, 2 voll.; deuxième édition Galatina, Congedo, 1990.

(26) Naudzana et Patiniana ou singularités remarquables, prises des conversations de MM. Naudé et Patin, [par Lancelot], Paris, Florentin et Pierre Delaulne, MDCCI. Exemplaire gardé à la Bibliothèque nationale de France sous la cote $\mathrm{Z}$ Payen 1138 , pp. 38-39 et 115-117. 
Tout comme Vanini, les écrivains-philosophes libertins ont dissimulé la transgression de leurs idées en s'appuyant sur une écriture qui présente deux niveaux de signification l'exotérique et l'ésotérique: le premier concerne le sens explicite du discours, tandis que le deuxième renvoie au signifié qui est caché au-dessous de la lettre. Ainsi, obtenus par un constant recours à toute sorte de ruse rhétorique (ironie, sousentendus, allusions, métaphores, périphrases, circonlocutions) et de ruse formelle (emploie du dialogue et utilisation de l'intertextualité), ces deux plans de signification ont donné naissance à une technique discursive, basée sur un équilibre précaire entre ce qui est dit et ce qui est sous-entendu, qui engendre des sens ambigus ${ }^{27}$.

L'influence de Vanini sur le courant libertin témoigne d'une continuité de pensée entre le $\mathrm{XVI}^{\mathrm{e}}$ et le $\mathrm{XVII}^{\mathrm{e}}$ siècle et en particulier entre la spéculation italienne de l'École padouane et le libertinisme. La présence de la culture italienne dans la culture française, en effet, va bien au-delà du Rinascimento car, comme le démontrent des études contemporaines qui s'appuient sur une documentation riche et variée, elle s'est épanouie aussi au siècle suivant ${ }^{28}$. Tout au long du XVII ${ }^{e}$ siècle, les nombreux voyages des Français en Italie et des Italiens en France sont à la base des influences réciproques entre ces deux pays: «hommes de science, érudits, voyageurs, poètes, religieux tous participent de ce dialogue privilégiés ${ }^{29}$. Un courant d'italianisme traverse cette période et joue un rôle important dans la formation intellectuelle des écrivains français ${ }^{30}$. Les innombrables traductions de l'italien en français effectuées pendant tout ce siècle ${ }^{31}$, d'ailleurs, témoignent que, comme à l'époque de la Renaissance, au $\mathrm{XVII}^{\mathrm{e}}$ siècle aussi, la culture française «cherche en Italie» ${ }^{32}$ et adapte à ses propres exigences quelques-unes des solutions trouvées. Le XVII siècle se confirme, par conséquent, un "siècle-laboratoire" "33, voire un "siècle-phare"34, où se croisent des cultures diverses et des dialogues multiples ${ }^{35}$.

MARCELLA LEOPIZZI

(27) On ne peut plus croire à la transparence du langage. Le signe et le sens s'éloignent. Le philosophe cherche d'autres voies pour dire sa vision du monde. Il procède par fables et fictions. Cfr. Jacques Prévost, Libertins du XVII siècle, Paris, Gallimard, 1998-2000, p. LXVII. Pour des approfondissements voir les livres suivants: Giulio Cesare Vanini e il libertinismo, actes du colloque de Taurisano (Lecce), 28-30 octobre 1999, par Francesco Paolo Raimondi, Galatina, Congedo, 2000; Giulio Cesare Vanini dal tardo Rinascimento al libertinisme érudit, actes du colloque de Taurisano (Lecce), 2426 octobre 1985, par Francesco Paolo RAIMONDI, Galatina, Congedo, 2003.

(28) Cfr. Lionello Sozzi, introduction à L'italianisme en France au XVII ${ }^{e}$ siècle, actes du $\mathrm{VIII}^{e}$ congrès de la Société Française de Littérature Comparée, (Grenoble-Chambéry, 26-28 mai 1966) recueillis et publiés par Giorgio Mirandola, Torino, Società Editrice Internazionale, 1968, pp. 7-9.

(29) Vito Castiglione Minischetti, Giovanni Dotoli, Paola Placella Sommella, Valeria PomPEJANO, Les traductions de l'italien en français au XVII siècle, Fasano-Paris, Schena-PUPS, 2001, quatrième de couverture.
(30) Cfr. Cecilia RizzA, «État présent des études sur les rapports franco-italiens au XVII ${ }^{\mathrm{e}}$ siècle», in L'italianisme en France au XVII siècle, cit., pp. 1119. Cfr. aussi Cecilia RizzA, «Libertins, libertinage, libertinismo: problema e prospettive», in Il Seicento francese oggi, actes du colloque international de Monopoli (Bari), 27-29 mai 1993, "Quaderni del Seicento francese”, cit., pp. 71-80.

(31) Voir à ce propos l'ouvrage suivant: MINISchetti, Dotoli, Placella Sommella, Pompejano, Les traductions de l'italien en français au XVII siècle, cit.

(32) LIONELLO SozzI, introduction à L'italianisme en France au XVII siècle, cit. p. 9.

(33) Giovanni Dotoli, «Perspectives de la recherche sur le XVII siècle français aujourd'hui», in Il Seicento francese oggi, atti del convegno internazionale di Monopoli 27-29 maggio 1993, "Quaderni del Seicento francese”, cit., pp. 25-59.

(34) Ibid.

(35) Cfr. Vito Castiglione Minischetti, GiovanNi Dotoli, Roger Musnik, Bibliographie $d u$ voyage français en Italie du Moyen Age à 1914, Fasano-Paris, Schena-Presses de l'Université de ParisSorbonne, 2002, introduction. 